\title{
Wake-Up Stroke: Clinical and Neuroimaging Characteristics
}

\author{
Gisele S. Silva ${ }^{a}$ e Fabricio O. Lima ${ }^{a}$ Erica C.S. Camargo ${ }^{a}$ Wade S. Smith ${ }^{c}$ \\ Aneesh B. Singhal ${ }^{a}$ David M. Greer ${ }^{a}$ Hakan Ay ${ }^{b}$ Michael H. Lev ${ }^{b}$ \\ Gordon J. Harris $^{b}$ Elkan F. Halpern ${ }^{b}$ Shruti Sonni ${ }^{a}$ Walter Koroshetz ${ }^{d}$ \\ Karen L. Furie ${ }^{a}$
}

Departments of a Neurology and ${ }^{b}$ Radiology, Massachusetts General Hospital and Harvard Medical School, Boston, Mass., 'Department of Neurology, University of California at San Francisco, San Francisco, Calif., and d National Institute of Neurological Disorders and Stroke, Bethesda, Md., USA; ${ }^{\mathrm{e}}$ Department of Neurology and Neurosurgery, Federal University of São Paulo, São Paulo, Brazil

\section{Key Words}

Wake-up stroke - Computed tomography angiography • Computed tomography perfusion $\cdot$ Ischemic penumbra

\begin{abstract}
Background: Approximately $25 \%$ of ischemic stroke patients awaken with neurological deficits. In these patients, in whom the time from symptom onset is uncertain, brain imaging is a potential strategy to characterize the ischemia duration and the presence of salvageable brain tissue. Methods: We prospectively evaluated consecutive patients with acute ischemic stroke. CT angiography and CT perfusion (CTP) were performed in patients within $24 \mathrm{~h}$ of symptom onset. The patients were classified into 'known onset', 'indefinite onset but not on awakening' and 'wake-up stroke' groups. Results: Of 676 patients evaluated, 420 had knownonset strokes, 131 wake-up strokes and 125 strokes with an indefinite time of symptom onset. Ischemic lesion volumes were higher in patients with indefinite-onset strokes $(p=$ 0.04). The frequencies of CTP mismatch and of large-vessel intracranial occlusions were similar among the groups $(p=$ 0.9 and $p=0.2$, respectively). Conclusion: The considerable prevalence of CTP mismatch and of intracranial artery occlu-
\end{abstract}

sions in our patients with wake-up strokes suggests that arterial and perfusion imaging might be particularly important in this population. Revised indications for thrombolysis by using imaging-based protocols might offer these patients the prospect of receiving acute stroke treatment even without a clear time of symptom onset.

Copyright $\odot 2010$ S. Karger AG, Basel

\section{Introduction}

Approximately $25 \%$ of patients with ischemic stroke awaken with neurological deficits [1]. Although some of these patients might be good candidates to receive acute treatment, they are not eligible for thrombolysis because of the uncertain time of stroke onset [2]. A retrospective analysis of the off-label use of thrombolytic therapy in wake-up strokes did not show an increased risk of intracranial bleeding, suggesting that at least a subset of those patients could benefit from reperfusion therapies [3]. Conversely, a randomized controlled trial of abciximab in acute ischemic stroke led to a higher risk of hemorrhagic transformation in patients with wake-up strokes than in patients with a definite onset time [4]. Therefore,

\section{KARGER}

Fax +41 613061234 E-Mail karger@karger.ch www.karger.com

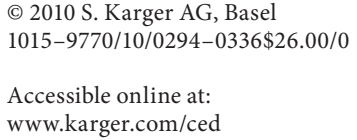

741 Altino Arantes Avenida apt 8

São Paulo, SP 04042-033 (Brazil)

Tel. +55 118358 0583, Fax +55 1150832172

E-Mail giselesampaio@hotmail.com 
if reperfusion therapies are to be used in patients with wake-up stroke, it is important to carefully select patients that more likely will benefit from treatment.

Neuroimaging techniques may allow an estimation of potential benefits and risks of therapies aiming at restoring perfusion [5]. Ischemic lesion volumes, and the presence of intracranial artery occlusions and of potentially salvageable brain tissue are neuroimaging characteristics that might influence the decision to treat ischemic stroke patients with reperfusion therapies [6]. Knowledge of the volume of the ischemic lesion might help estimate the risk for hemorrhagic transformation after reperfusion because lesions with higher volumes are more prone to hemorrhagic transformation [7]. In addition to detecting the presence of intracranial artery occlusions, CT angiography (CTA) can estimate ischemic lesion volumes via an analysis of CTA source images (CTA-SI). CTA-SI correlate with lesion volumes on diffusion-weighted imaging and may have advantages over other techniques because CTASI cover the entire brain and are available at the completion of imaging [8]. The perfusion/diffusion mismatch is a surrogate marker of salvageable brain tissue and of the time of stroke onset, considering that the amount of mismatch declines with the time from symptom onset [9]. The CT perfusion (CTP) cerebral blood flow/cerebral blood volume (CBF/CBV) mismatch is comparable to the perfusion/diffusion mismatch for the evaluation of cerebral penumbra in acute ischemic stroke [8].

In wake-up strokes, findings on CTA and CTA-SI, and the prevalence of CTP mismatch have not been studied. Our objective was to describe the clinical, CTA, CTA-SI and CTP characteristics of patients with wake-up strokes, and to compare these findings with those of patients with a known time of onset and indefinite onset time due to the lack of a witness.

\section{Patients and Methods}

We evaluated consecutive patients enrolled in a prospective cohort study at 2 university-based hospitals from March 2003 to December 2005. Noncontrast CT (NCCT) scans, CTA and CTASI were performed for suspected ischemic stroke within $24 \mathrm{~h}$ from symptom onset or from last seen well. Patients with intracranial hemorrhage or an alternative cause of the neurological symptoms, and those in whom iodinated contrast administration was contraindicated (i.e. contrast allergy, pregnancy, congestive heart failure and glomerular filtration rate $<30 \mathrm{ml} / \mathrm{min}$ ) were excluded. CTP was performed within $12 \mathrm{~h}$ of symptom onset or last seen well unless the treating neurologist contraindicated the additional dose of contrast. CT, CTA and CTP were performed at the same session for each patient. The data collected included demograph- ics, National Institute of Health Stroke Scale (NIHSS) scores, modified Rankin Scale (mRS) scores before stroke, at discharge and at 6 months, Stop Stroke Study-TOAST (SSS-TOAST) and Oxfordshire community stroke project classifications, neuroimaging characteristics and thrombolysis status [10]. The patients were classified into 3 groups based on the circumstance of symptom identification: known-onset strokes (recognized by the patient or by a witness), indefinite-onset strokes but not on awakening (unknown onset time due to the lack of a witness when the patient was unable to determine the time of symptom onset), and wake-up strokes (symptoms on awakening recognized by a witness or by the patient) [11]. The prospective study received institutional review board approval. All participants provided their written informed consent.

\section{Neuroimaging Protocol}

NCCT and CTA acquisitions were performed with 16- or 64section multidetector CT scanners (Lightspeed; GE Healthcare, Milwaukee, Wisc., USA). Representative sample parameters were $120-140 \mathrm{kVp}, 170 \mathrm{~mA}$, scan time $=2 \mathrm{~s}$ and section thickness $=$ $5 \mathrm{~mm}$ for NCCT. CTA was performed $25 \mathrm{~s}$ after the intravenous administration of 100-140 ml of a nonionic contrast agent (Isovue; Bracco Diagnostics, Princeton, N.J., USA) by power injector at $3 \mathrm{ml} / \mathrm{s}$ (Medrad Power Injector; Medrad, Indianola, Pa., USA) [8]. The parameters were $140 \mathrm{kVp}, 220-250 \mathrm{~mA}$, rotation time = $0.8-1.0 \mathrm{~s}$, section thickness $=2.5 \mathrm{~mm}$, reconstruction interval $=$ $1.25 \mathrm{~mm}, 3.75 \mathrm{~mm}$ per rotation table speed and 0.75:1 pitch. Immediately afterward, a second set of images was obtained from the aortic arch to the skull base. Source images were reconstructed into standardized maximum intensity projection views of the intracranial and extracranial vasculature.

CTP was initiated $5 \mathrm{~s}$ after the administration of $45 \mathrm{ml}$ of contrast at $7 \mathrm{ml} / \mathrm{s}$. Four contiguous, 5 -mm-thick CT slices were acquired simultaneously every second, during 45-60 s. The postprocessing of $\mathrm{CBF}$ and $\mathrm{CBV}$ maps was done using the software $\mathrm{CT}$ Perfusion 3 (GE Healthcare) [8].

The images were analyzed by consensus by a neuroradiologist and 2 neurologists experienced in stroke neuroimaging. The reviewers were blinded to the patients' clinical characteristics and to the CTA-SI and follow-up CT studies performed for each patient when evaluating the presence of a $\mathrm{CBF} / \mathrm{CBV}$ mismatch. Brain magnetic resonance imaging (MRI) was performed as indicated on clinical grounds in selected patients, but it was not a part of the research protocol and was not available to the reviewers when CT, CTA and CTP were being evaluated. Axial CTA-SI were evaluated for ischemic regions by identifying areas of relative hypoattenuation. Using a semiautomated software (Alice; Parexel, Waltham, Mass., USA), these regions were visually segmented to determine the total lesion volume [12]. CTA was used to assess for arterial stenoses and occlusions. Large intracranial vessel occlusions amenable to intra-arterial neurointerventional approaches were defined as middle cerebral artery (M1 or proximal M2), internal carotid artery or basilar occlusions when those arteries were the culprits in the acute stroke [13]. Hemorrhagic transformation was defined as any apparently extravascular blood in the brain or within the cranium, and was classified as symptomatic if associated with clinical deterioration as defined by an increase of 4 points or more on the NIHSS [14].

CTP was analyzed for the presence of a CBF/CBV mismatch by visual inspection. A mismatch was defined as at least $25 \%$ larg- 
er perfusion abnormality (measured by $\mathrm{CBF}$ ) compared with the CBV deficit. CTP scans that did not cover the maximal area of the expected perfusion abnormality were excluded. Additionally, to avoid overestimating perfusion deficits due to bolus-tracking time delay, we excluded CTP of patients with atrial fibrillation and of patients with cervical internal carotid artery occlusions ipsilateral to the stroke.

\section{Statistical Analysis}

Means and SD, or medians and interquartile intervals were used to describe the patients' characteristics. One-way ANOVA and the Kruskal-Wallis test were used for the comparison of continuous parametric and nonparametric variables, respectively. The Mann-Whitney test with Bonferroni correction was used to compare the different groups against the definite-time group. Categorical comparisons were made by the $\chi^{2}$ or Fisher exact test. We used logistic regression analysis to investigate the influence of the circumstance of stroke symptom identification (known onset, indefinite onset and wake-up stroke) upon 6 months mRS scores dichotomized at 2 ( $\mathrm{mRS} \leq 2 \mathrm{vs}$. $>2)$, with adjustment for age, sex, prestroke mRS score, NIHSS score at admission, volume of ischemic lesion, and thrombolytic therapy. $\mathrm{p}<0.05$ was considered significant. SPSS (SPSS, Chicago, Ill., USA) was used for the calculations.

\section{Results}

Of 676 patients evaluated from March 2003 to December 2005, 420 patients had known-onset, 125 indefinite-onset and 131 wake-up strokes. The known-onset and wake-up stroke groups did not differ significantly in age, gender, NIHSS score at admission or SSS-TOAST subtype. Patients with indefinite-onset strokes were predominantly female, older and had higher NIHSS scores at admission (table 1). The time interval from stroke symptom onset (or from last seen well) to first neuroimaging was longer in patients with wake-up and indefinite-onset strokes compared to those with knownonset stroke. Twenty percent of the patients had posterior circulation strokes, $16.8 \%$ total anterior circulation strokes, $44.4 \%$ partial anterior circulation strokes and $18.8 \%$ lacunar strokes. There was no difference in the frequency of Oxfordshire classification subtypes between the 3 different groups of stroke symptom recognition $(\mathrm{p}=0.42)$.

CTA and CTA-SI were available for all patients. The large vessel intracranial occlusions amenable to intra-arterial neurointerventional approaches (M1 and proximal M2, internal carotid artery or basilar arteries) were similar among the 3 groups (fig. 1; table 2). The CTA-SI lesion volumes were higher in patients with indefinite-onset strokes than in patients with known-onset strokes. There were no differences in ischemic lesion volumes between the patients with wake-up and known-onset strokes (table 1; fig. 2).

A total of 393 patients (58\%) underwent CTP at baseline. We excluded 186 CTP from the analysis due to lack of coverage of the maximal area of the expected perfusion abnormality, 32 due to atrial fibrillation at admission and 7 due to cervical internal carotid artery occlusions ipsilateral to the stroke. The frequency of CTP exams was similar among the 3 groups both before and after excluding poor-quality exams ( $p=0.2$ and $p=0.3$, respectively). In the $170 \mathrm{CTP}$ evaluated, the frequency of $\mathrm{CBF} / \mathrm{CBV}$ mismatch was not significantly different among the groups (table 1; fig. 1).

The patients with indefinite-onset or wake-up strokes who presented within a 3-hour window from last seen well and were eligible received intravenous tissue plasminogen activator. An 8-hour window was used for intraarterial approaches to revascularization. Patients with known onset were more frequently treated with intravenous tissue plasminogen activator $(\mathrm{p}<0.01)$. There was no difference in the frequency of symptomatic hemorrhagic transformations between the 3 different groups of stroke symptom recognition (table 1). The patients with indefinite-onset strokes had a higher frequency of asymptomatic hemorrhagic transformations when compared to patients with known-onset strokes $(\mathrm{p}<0.01)$. In the patients treated with intravenous thrombolysis, there was no difference in the frequency of symptomatic or asymptomatic hemorrhagic transformations between the 3 groups.

The patients with indefinite onset had the poorest outcomes at discharge, with only $32 \%$ achieving an mRS score of $0-2$, compared to $48 \%$ in the other groups ( $\mathrm{p}<$ 0.01) (fig. 1). At 6 months, after adjustment for age, sex, prestroke mRS, NIHSS score at admission, the volume of ischemic lesion, and thrombolytic therapy, the circumstance of stroke symptom identification was no longer a predictor of $\mathrm{mRS}$ scores $(\mathrm{p}=0.38)$. Treatment with intravenous thrombolysis, male sex, lower prestroke mRS scores, stroke volumes and NIHSS scores at admission were all predictors of good functional outcome (mRS scores of $0-2$ ) at 6 months.

\section{Discussion}

In the present study, we found that patients with wakeup and known-onset strokes were clinically similar. Patients with indefinite onset, however, had more severe deficits at admission, different clinical characteristics and a worse prognosis at discharge. 
Table 1. Patient characteristics

\begin{tabular}{|c|c|c|c|c|}
\hline & $\begin{array}{l}\text { Known } \\
\text { onset time } \\
(n=420)\end{array}$ & $\begin{array}{l}\text { Indefinite } \\
\text { onset time } \\
(\mathrm{n}=125)\end{array}$ & $\begin{array}{l}\text { Wake-up } \\
\text { strokes } \\
(\mathrm{n}=131)\end{array}$ & $\mathrm{p}$ \\
\hline Females, \% & 44 & $57^{*}$ & 48 & 0.04 \\
\hline Age, years & $67.5 \pm 15.2$ & $71 \pm 14.9^{*}$ & $68.8 \pm 15.4$ & 0.08 \\
\hline Median NIHSS score & $5(2-11)$ & $8(3-16)^{*}$ & $5(2-11)$ & $<0.01$ \\
\hline Hypertension, \% & 58.1 & $71.2^{*}$ & 63.4 & 0.03 \\
\hline Diabetes, $\%$ & 18.3 & 19.2 & 16.8 & 0.88 \\
\hline Hyperlipidemia, \% & 30.0 & 28.2 & 22.9 & 0.26 \\
\hline Atrial fibrillation, $\%$ & 20.2 & 29.6 & 19.1 & 0.06 \\
\hline Coronary artery disease, $\%$ & 24 & 22.4 & 20.6 & 0.70 \\
\hline Arterial pressure at admission, $\mathrm{mm} \mathrm{Hg}$ & $104.9 \pm 18.3$ & $104.4 \pm 17.2$ & $107.9 \pm 16.4$ & 0.10 \\
\hline Glucose at admission, mg/dl & $134 \pm 55.8$ & $142 \pm 58.5$ & $132 \pm 46.4$ & 0.10 \\
\hline \multicolumn{5}{|l|}{ SSS-TOAST classification ${ }^{1}, \%$} \\
\hline Cardioembolic $^{1}$ & 38.4 & 45 & 36.2 & \multirow[t]{5}{*}{0.30} \\
\hline Large vessel $^{1}$ & 15.9 & 19.2 & 16.1 & \\
\hline Small vessel ${ }^{1}$ & 8.9 & 6.7 & 13.8 & \\
\hline Undetermined $^{2}$ & 29 & 23.3 & 28.5 & \\
\hline Other ${ }^{1}$ & 7.8 & 5.8 & 5.4 & \\
\hline Time to first neuroimaging ${ }^{3}, \mathrm{~h}$ & $5.7 \pm 7.2$ & $8.2 \pm 7.9^{*}$ & $12.4 \pm 8.8^{*}$ & $<0.01$ \\
\hline Intravenous thrombolysis, $\%$ & 22.3 & $14.4^{*}$ & $2.3^{*}$ & $<0.01$ \\
\hline Intra-arterial thrombolysis ${ }^{4}, \%$ & 5.8 & 8.8 & 1.5 & 0.03 \\
\hline Asymptomatic intracranial hemorrhage $e^{5}, \%$ & 1.4 & $5.6^{*}$ & 3.1 & 0.03 \\
\hline Symptomatic intracranial hemorrhage ${ }^{5}, \%$ & 1.7 & 1.6 & 1.5 & 0.99 \\
\hline Median CTA-SI lesion volume, $\mathrm{ml}$ & $14.29(0-137.36)$ & $46.55(0-220.83)^{*}$ & $14.35(0-217.27)$ & 0.04 \\
\hline $\mathrm{CBF} / \mathrm{CBV}$ mismatch, \% & 37.0 & 40.7 & 37.1 & 0.93 \\
\hline \multicolumn{5}{|c|}{ entheses denote interquartile rang } \\
\hline \multirow{2}{*}{\multicolumn{5}{|c|}{$\begin{array}{l}0.025 \text { when compared to the reference category (known-onset stroke) after Bonferroni correction. } \\
{ }^{1} \text { Definite, probable and possible categories are shown together. }\end{array}$}} \\
\hline & & & & \\
\hline \multicolumn{5}{|c|}{${ }^{2}$ Unknown, cryptogenic emboli and $>1$ mechanism. } \\
\hline \multicolumn{5}{|l|}{${ }^{3}$ From symptom onset or last seen well. } \\
\hline \multirow{2}{*}{\multicolumn{5}{|c|}{$\begin{array}{l}{ }^{4} \text { Includes } 36 \text { patients ( } 28 \text { patients treated with intra-arterial drugs only, } 5 \text { treated with mechanical clot re- } \\
\text { moval only, and } 3 \text { treated with mechanical clot removal in addition to intra-arterial drugs). }\end{array}$}} \\
\hline & & & & \\
\hline \multicolumn{5}{|c|}{$\begin{array}{l}\text { moval only, and } 3 \text { treated with mechanical clot removal in addition to intra-arterial drugs). } \\
{ }^{5} \text { Frequency of hemorrhagic transformation for all patients. In patients treated with intravenous thrombol- }\end{array}$} \\
\hline
\end{tabular}

Patients with indefinite-onset strokes, but not those with wake-up strokes, had higher volumes of ischemic lesion when compared to patients with known-onset strokes. The frequency of $\mathrm{CBF} / \mathrm{CBV}$ mismatch, however, was similar, irrespective of the circumstance of stroke symptom identification. In acute ischemic stroke, ischemic lesion volume grows with time from symptom onset and might therefore be used as a marker of ischemia duration [15]. Although the exact time of symptom onset was unclear for both wake-up and indefinite-onset strokes, in patients with wake-up strokes less time might have elapsed from the onset of stroke. Circadian variation is well described for strokes, with the most cerebrovascu- lar events witnessed happening during the morning [16]. It is possible that some patients who wake up with symptoms had their stroke onset close to the time of arousal and could therefore benefit from reperfusion therapies.

The progression of ischemic lesions into the penumbra area has been described to occur beyond $3 \mathrm{~h}$ of symptom onset $[17,18]$. Furthermore, the reperfusion of an area of initial hypoperfusion has been associated with a reduced infarct growth and favorable clinical outcome $[17,18]$. Consequently, neuroimaging is a potentially powerful tool to identify patients with uncertain onset time that could possibly benefit from thrombolysis. Since the frequency of mismatch was similar in the 3 groups, and mis- 


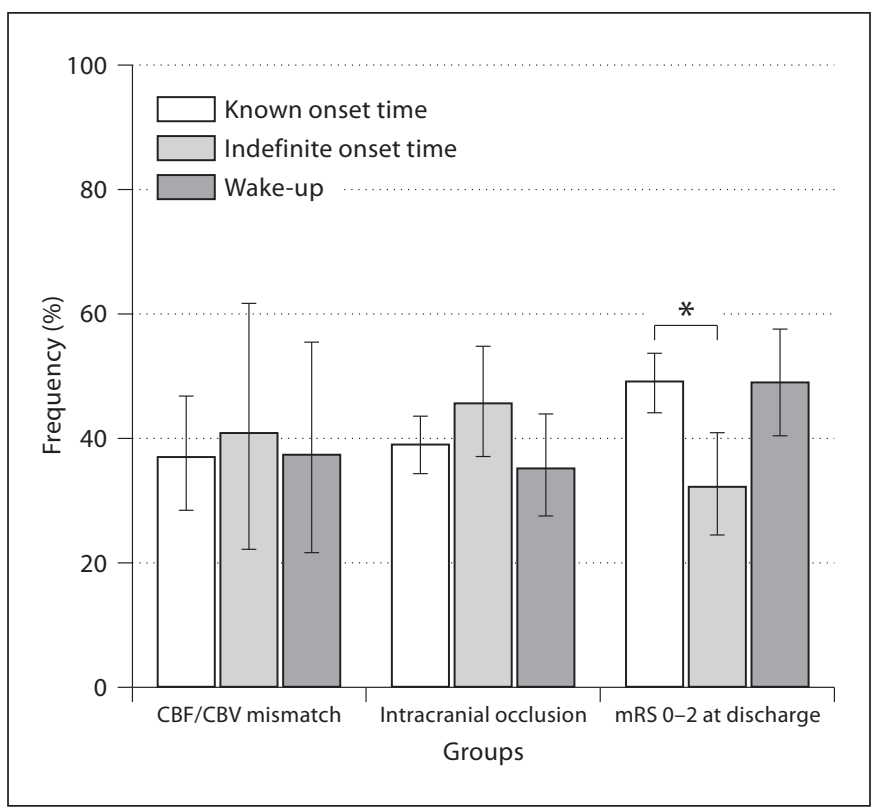

Fig. 1. Frequency of patients with $\mathrm{CBF} / \mathrm{CBV}$ mismatch, intracranial occlusions amenable to neurointerventional approaches on CTA, and an mRS score of $0-2$ at discharge in each group. ${ }^{*} \mathrm{p}<$ 0.025 when compared to the reference category (known-onset strokes) after Bonferroni correction. Error bars: 95\% CI.

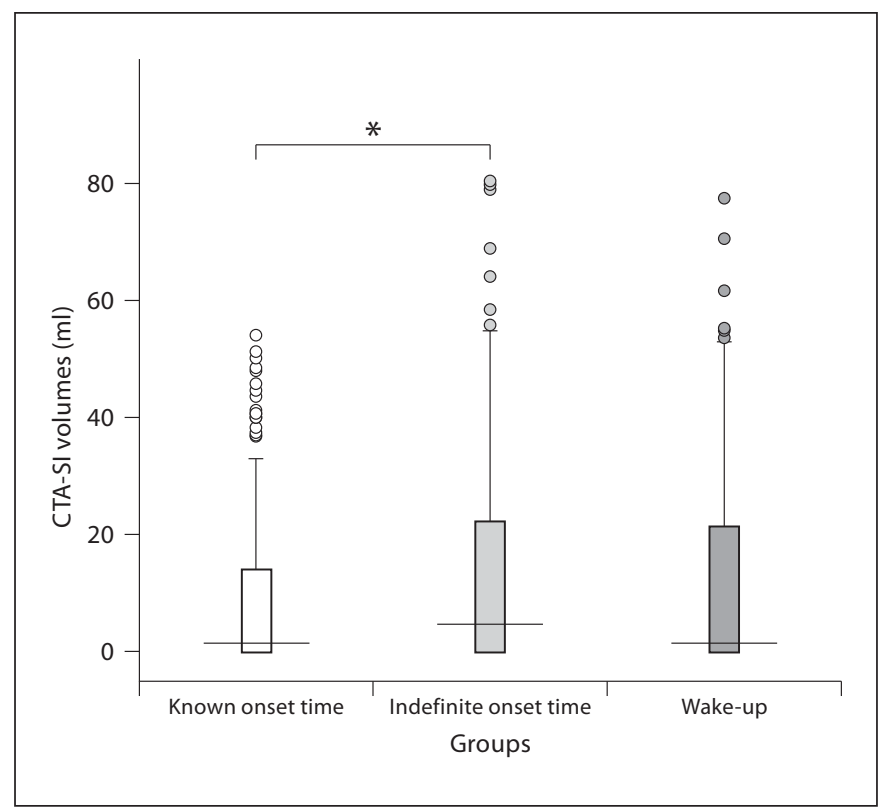

Fig. 2. CTA-SI ischemic volumes in the 3 stroke groups. $50 \%$ of the values are within the box, $25 \%$ are higher and 25\% lower than the values within the box. Horizontal line inside the box: median. Upper boundary of whisker: highest observed value that is not an outlier. Lower boundary of whisker: lowest observed value that is not an outlier. Circles: outliers.

Table 2. Intracranial occlusions on CTA in the 3 stroke groups

\begin{tabular}{|c|c|c|c|c|}
\hline & $\begin{array}{l}\text { Known } \\
\text { onset time } \\
(n=420)\end{array}$ & $\begin{array}{l}\text { Indefinite } \\
\text { onset time } \\
(\mathrm{n}=125)\end{array}$ & $\begin{array}{l}\text { Wake-up } \\
\text { strokes } \\
(\mathrm{n}=131)\end{array}$ & $\mathrm{p}$ \\
\hline Any intracranial occlusion ${ }^{1}$ & $38.6 \%$ & $45.6 \%$ & $35.1 \%$ & 0.20 \\
\hline Middle cerebral artery M1 & $24.0 \%$ & $20.7 \%$ & $14.5 \%$ & 0.14 \\
\hline Middle cerebral artery M2 & $21.7 \%$ & $19.2 \%$ & $16.8 \%$ & 0.20 \\
\hline Internal carotid artery & $27.9 \%$ & $28.0 \%$ & $19.8 \%$ & 0.17 \\
\hline T occlusion & $2.1 \%$ & $2.4 \%$ & $0.8 \%$ & 0.55 \\
\hline Basilar & $1.4 \%$ & $4.0 \%$ & $4.6 \%$ & 0.06 \\
\hline
\end{tabular}

${ }^{1} \mathrm{M} 1$, proximal M2, internal carotid artery (including T lesions) or basilar occlusions. Some patients had occlusions of $>1$ artery.

match is a surrogate marker of tissue viability, it can be inferred that a similar proportion of patients from all 3 groups had ischemic penumbra potentially salvageable by reperfusion [18]. Nevertheless, the risk/benefit ratio of thrombolytic therapy would likely be unfavorable to indefinite-onset patients because of the larger ischemic lesions and consequently increased risk of hemorrhagic transformation and poor clinical outcome [7]. In fact, as- ymptomatic hemorrhagic transformation was more common in patients with an indefinite onset time in our series. Possible explanations for this finding include greater ischemic volumes in this group, higher rates of treatment with intra-arterial thrombolysis and, likely, more time elapsed from stroke onset. Our results are in accordance with those from the study of Fink et al. [5], which demonstrated, by using MRI, that a similar pro- 
portion of known-onset and wake-up strokes have imaging parameters compatible with the presence of salvageable tissue. Other authors showed that patients with indefinite stroke onset time, even when evaluated within $3 \mathrm{~h}$ of stroke recognition, are more likely to have definite hypodense areas on NCCT when compared to wake-up and known-onset strokes [11].

Wake-up strokes had outcomes at hospital discharge similar to those of known-onset strokes despite the higher percentage of patients treated with thrombolytics in known-onset strokes. Although similar outcomes in wake-up and known-onset strokes have been previously reported, a recent randomized controlled trial showed that abciximab can lead to a worse prognosis in wake-up strokes when compared to known-onset strokes [19]. The administration of abciximab, however, was not based on neuroimaging characteristics with a favorable risk/benefit ratio for reperfusion therapies [4]. In our study, the poorer outcomes of patients with indefinite-onset strokes when compared to patients with known-onset strokes were explained by more severe deficits at admission (higher NIHSS scores), older age, female sex, larger ischemic lesion volumes and a lower frequency of treatments with intravenous thrombolytic therapy, all predictors of outcome following ischemic stroke in other series [20].

This study has a number of limitations. First, CTP was performed in a subset of approximately $60 \%$ of the patients. Patients who did not have a CTP done, however, were clinically similar to patients evaluated by CTP, except that most were more than $12 \mathrm{~h}$ from symptom onset or from last seen well, because CTP was routinely performed only within $12 \mathrm{~h}$ of stroke symptom onset. The similar frequency of CTP exams among the 3 groups, however, suggests that there was no systematic bias regarding groups. Therefore, our CTP data are likely generalizable to the subset of patients who present to the hos- pital within $12 \mathrm{~h}$ from symptom onset or from last seen well. Secondly, the presence of a CTP mismatch suggests, but does not prove, tissue viability [21]. Although there is good evidence that patients with MRI perfusion/diffusion-weighted imaging mismatch at presentation seem to benefit from reperfusion therapies, a definitive study in which the therapeutic window is based on the presence of mismatch only, and not on the time from symptom onset, is still lacking [18]. The ideal definition and measurement of mismatch using CTP and MRI are not completely established. Perfusion imaging parameters which indicate that tissue is at risk for infarction and could potentially have clinical relevance or that adequate reperfusion has occurred are still undetermined as well [22]. Therefore, we cannot extrapolate the safety or efficacy of reperfusion therapies in patients with wake-up strokes based on our data. We used only visual inspection in order to determine mismatch presence. Although quantitative methods would be ideal to measure the extent of ischemic penumbra, visual inspection is practical in the acute setting and more reflective of real life acute stroke practice [23].

The considerable prevalence of CTP mismatch and of intracranial artery occlusions in our patients with wakeup strokes suggests that arterial and perfusion imaging might be particularly important in this population. Revised indications for thrombolysis, using imaging-based protocols, might offer these patients the prospect of receiving acute stroke treatment even without a clear time of symptom onset [6].

\section{Acknowledgment}

This study was supported by NIH grant AHRQ R01 HS11392 (K.L.F.)

\section{References}

1 Nadeau JO, Fang J, Kapral MK, Silver FL, Hill MD: Outcome after stroke upon awakening. Can J Neurol Sci 2005;32:232-236.

-2 National Institute of Neurological Disorders and Stroke rt-PA Stroke Study Group: Tissue plasminogen activator for acute ischemic stroke. N Engl J Med 1995;333:1581-1587.

-3 Barreto AD, Martin-Schild S, Hallevi H, Morales MM, Abraham AT, Gonzales NR, Illoh K, Grotta JC, Savitz SI: Thrombolytic therapy for patients who wake up with stroke. Stroke 2009; 40:827-832.
-4 Adams HPJ, Leira EC, Torner JC, Barnathan E, Padgett L, Effron MB, Hacke W: Treating patients with 'wake-up' stroke: the experience of the AbESTT-II trial. Stroke 2008;39: 3277-3282.

5 Fink JN, Kumar S, Horkan C, Linfante I, Se$\lim \mathrm{MH}$, Caplan LR, Schlaug G: The stroke patient who woke up: clinical and radiological features, including diffusion and perfusion MRI. Stroke 2002;33:988-993.
6 Cho A, Sohn S, Han M, Lee DH, Kim JS, Choi CG, Sohn C, Kwon SU, Suh DC, Kim SJ, Bae H, Kang D: Safety and efficacy of MRI-based thrombolysis in unclear-onset stroke: a preliminary report. Cerebrovasc Dis 2008;25: 572-579.

7 Lansberg MG, Thijs VN, Bammer R, Kemp S, Wijman CAC, Marks MP, Albers GW: Risk factors of symptomatic intracerebral hemorrhage after tPA therapy for acute stroke. Stroke 2007;38:2275-2278. 
8 Schaefer PW, Barak ER, Kamalian S, Gharai LR, Schwamm L, Gonzalez RG, Lev MH: Quantitative assessment of core/penumbra mismatch in acute stroke: CT and MR perfusion imaging are strongly correlated when sufficient brain volume is imaged. Stroke 2008;39:2986-2992.

-9 Darby DG, Barber PA, Gerraty RP, Desmond PM, Yang Q, Parsons M, Li T, Tress BM, Davis SM: Pathophysiological topography of acute ischemia by combined diffusionweighted and perfusion MRI. Stroke 1999; 30:2043-2052.

-10 Ay H, Benner T, Arsava EM, Furie KL, Singhal $\mathrm{AB}$, Jensen $\mathrm{MB}$, Ayata $\mathrm{C}$, Towfighi A, Smith EE, Chong JY, Koroshetz WJ, Sorensen AG: A computerized algorithm for etiologic classification of ischemic stroke: the Causative Classification of Stroke System. Stroke 2007;38:2979-2984.

- 11 Todo K, Moriwaki H, Saito K, Tanaka M, Oe $\mathrm{H}$, Naritomi H: Early CT findings in unknown-onset and wake-up strokes. Cerebrovasc Dis 2006;21:367-371.

-12 Schwamm LH, Rosenthal ES, Swap CJ, Rosand J, Rordorf G, Buonanno FS, Vangel MG, Koroshetz WJ, Lev MH: Hypoattenuation on CT angiographic source images predicts risk of intracerebral hemorrhage and outcome after intra-arterial reperfusion therapy. AJNR Am J Neuroradiol 2005;26: 1798-1803.

13 Smith WS, Sung G, Saver J, Budzik R, Duckwiler G, Liebeskind DS, Lutsep HL, Rymer MM, Higashida RT, Starkman S, Gobin YP, Frei D, Grobelny T, Hellinger F, Huddle D, Kidwell C, Koroshetz W, Marks M, Nesbit G, Silverman IE: Mechanical thrombectomy for acute ischemic stroke: final results of the Multi MERCI trial. Stroke 2008;39:12051212 .
14 Hacke W, Kaste M, Fieschi C, von Kummer R, Davalos A, Meier D, Larrue V, Bluhmki E, Davis S, Donnan G, Schneider D, Diez-Tejedor E, Trouillas P: Randomised double-blind placebo-controlled trial of thrombolytic therapy with intravenous alteplase in acute ischaemic stroke (ECASS II). Second European-Australasian Acute Stroke Study Investigators. Lancet 1998;352:1245-1251.

15 Schwamm LH, Koroshetz WJ, Sorensen AG, Wang B, Copen WA, Budzik R, Rordorf G, Buonanno FS, Schaefer PW, Gonzalez RG: Time course of lesion development in patients with acute stroke: serial diffusionand hemodynamic-weighted magnetic resonance imaging. Stroke 1998;29:2268-2276.

16 Elliott WJ: Circadian variation in the timing of stroke onset: a meta-analysis. Stroke 1998; 29:992-996.

17 Albers GW, Thijs VN, Wechsler L, Kemp S, Schlaug G, Skalabrin E, Bammer R, Kakuda W, Lansberg MG, Shuaib A, Coplin W, Hamilton S, Moseley M, Marks MP: Magnetic resonance imaging profiles predict clinical response to early reperfusion: the diffusion and perfusion imaging evaluation for understanding stroke evolution (DEFUSE) study. Ann Neurol 2006;60:508-517.

18 Davis SM, Donnan GA, Parsons MW, Levi C, Butcher KS, Peeters A, Barber PA, Bladin C, de Silva DA, Byrnes G, Chalk JB, Fink JN, Kimber TE, Schultz D, Hand PJ, Frayne J, Hankey G, Muir K, Gerraty R, Tress BM, Desmond PM: Effects of alteplase beyond 3 $\mathrm{h}$ after stroke in the Echoplanar Imaging Thrombolytic Evaluation Trial (EPITHET) a placebo-controlled randomised trial. Lancet Neurol 2008;7:299-309.
19 Serena J, Dávalos A, Segura T, Mostacero E, Castillo J: Stroke on awakening: looking for a more rational management. Cerebrovasc Dis 2003;16:128-133.

20 Hankey GJ, Jamrozik K, Broadhurst RJ, Forbes S, Anderson CS: Long-term disability after first-ever stroke and related prognostic factors in the Perth Community Stroke Study, 1989-1990. Stroke 2002;33:10341040.

21 Schramm P, Schellinger PD, Klotz E, Kallenberg K, Fiebach JB, Külkens S, Heiland S, Knauth M, Sartor K: Comparison of perfusion computed tomography and computed tomography angiography source images with perfusion-weighted imaging and diffusion-weighted imaging in patients with acute stroke of less than 6 hours' duration. Stroke 2004;35:1652-1658.

-22 Wintermark M, Albers GW, Alexandrov AV, Alger JR, Bammer R, Baron JC, Davis S, Demaerschalk BM, Derdeyn CP, Donnan GA, Eastwood JD, Fiebach JB, Fisher M, Furie KL, Goldmakher GV, Hacke W, Kidwell CS, Kloska SP, Köhrmann M, Koroshetz W, Lee TY, Lees KR, Lev MH, Liebeskind DS, Ostergaard L, Powers WJ, Provenzale J, Schellinger P, Silbergleit R, Sorensen AG, Wardlaw J, Wu $\mathrm{O}$, Warach S: Acute stroke imaging research roadmap. Stroke 2008;39:1621-1628.

23 Wintermark M, Reichhart M, Cuisenaire O, Maeder P, Thiran J, Schnyder P, Bogousslavsky J, Meuli R: Comparison of admission perfusion computed tomography and qualitative diffusion- and perfusionweighted magnetic resonance imaging in acute stroke patients. Stroke 2002;33:20252031. 